\title{
microRNAs are ligands of Toll-like receptors
}

\author{
XI CHEN, ${ }^{1}$ HONGWEI LIANG, JUNFENG ZHANG, KE ZEN, and CHEN-YU ZHANG ${ }^{1}$ \\ Jiangsu Engineering Research Center for microRNA Biology and Biotechnology, State Key Laboratory of Pharmaceutical Biotechnology, School of Life \\ Sciences, Nanjing University, Nanjing 210093, China
}

\begin{abstract}
It has long been known that microRNAs (miRNAs) can regulate target gene expression at the post-transcriptional level. Recent studies, however, have revealed that miRNAs can also be transported from donor cells to recipient cells, in which these RNAs function in a novel manner as ligands of Toll-like receptors. Here, we review the latest findings on these unconventional miRNAs, with special emphasis on their biological significance.
\end{abstract}

Keywords: microRNA; Toll-like receptor; ligand; exosome

\section{INTRODUCTION}

MicroRNAs (miRNAs) are a class of naturally occurring small noncoding RNAs of 19-24 nt in length (Bartel 2004). It is common knowledge that miRNAs function via base-pairing with complementary sites on target mRNAs, thus causing either translational repression or direct mRNA degradation (Bartel 2004). However, recent studies suggest that miRNAs work in a much more sophisticated manner than initially assumed. For example, miRNAs can be secreted into the extracellular environment as signaling molecules to mediate intercellular communication (Chen et al. 2012b) or be transported from the cytoplasm to the nucleus to regulate their own expression (Chen et al. 2012a). More strikingly, recent studies indicate that miRNAs can even serve as physiological ligands for Toll-like receptors (TLRs), a function that is independent of their conventional role in post-transcriptional gene regulation (Fabbri et al. 2012; Lehmann et al. 2012). These discoveries reveal an intriguing and unusual working model of miRNAs.

\section{INTRACELLULAR TLRS AND THEIR FUNCTION AS SENSORS OF FOREIGN NUCLEIC ACIDS}

TLRs are a family of receptors through which the innate immune system of the host recognizes the presence of invading pathogens (Takeda et al. 2003; Blasius and Beutler 2010). To date, 10 human TLRs have been identified. TLR1, TLR2, TLR4, TLR5, TLR6, and TLR10 are expressed largely on the cell surface, where they recognize molecules derived from

${ }^{1}$ Corresponding authors

E-mail xichen@nju.edu.cn

E-mail cyzhang@nju.edu.cn

Article published online ahead of print. Article and publication date are at http://www.rnajournal.org/cgi/doi/10.1261/rna.036319.112. microbes (e.g., lipopolysaccharides) (Takeda et al. 2003; Blasius and Beutler 2010). The intracellular TLRs, including TLR3, TLR7, TLR8, and TLR9, are intrinsically capable of detecting foreign nucleic acids, including double- and singlestranded RNA (ssRNA) from RNA viruses and DNA from bacteria and DNA viruses (Takeda et al. 2003; Blasius and Beutler 2010). For example, it has been shown that murine TLR7 and human TLR8 mediate species-specific recognition of GU-rich ssRNA derived from human immunodeficiency virus-1 (HIV-1) (Heil et al. 2004). The nucleic acid-specific TLRs are located within endolysosomal compartments and can trigger the induction of cytokines essential for innate immune responses (Takeda et al. 2003; Blasius and Beutler 2010).

\section{miRNAs FUNCTION AS TLR LIGANDS IN TUMOR CELLS}

Recently, Fabbri et al. found that lung tumor cells can secrete miR-21 and miR-29a via exosomes, and these exosomes can be transferred from cell to cell and can be taken up by macrophages at the tumor-normal tissue interface and eventually reach TLR-containing endosomes (Fig. 1; Fabbri et al. 2012). Although previous studies have demonstrated that secreted miRNAs within exosomes can regulate gene expression in the recipient cells via canonical binding to their target mRNAs, this study showed that tumor-secreted miR-21 and miR-29a can function through another mechanism-by interacting with murine TLR7 and human TLR8 in the endosomes (Fig. 1; Fabbri et al. 2012). Importantly, the authors provided first evidence that miR-21 and miR-29a can directly bind to TLR8 by performing coimmunoprecipitation assays (Fabbri et al. 2012). The interaction between miR-21/29a and TLR7/8 may cause macrophages to secrete proinflammatory cytokines (e.g., TNF- $\alpha$ and IL-6), ultimately leading 


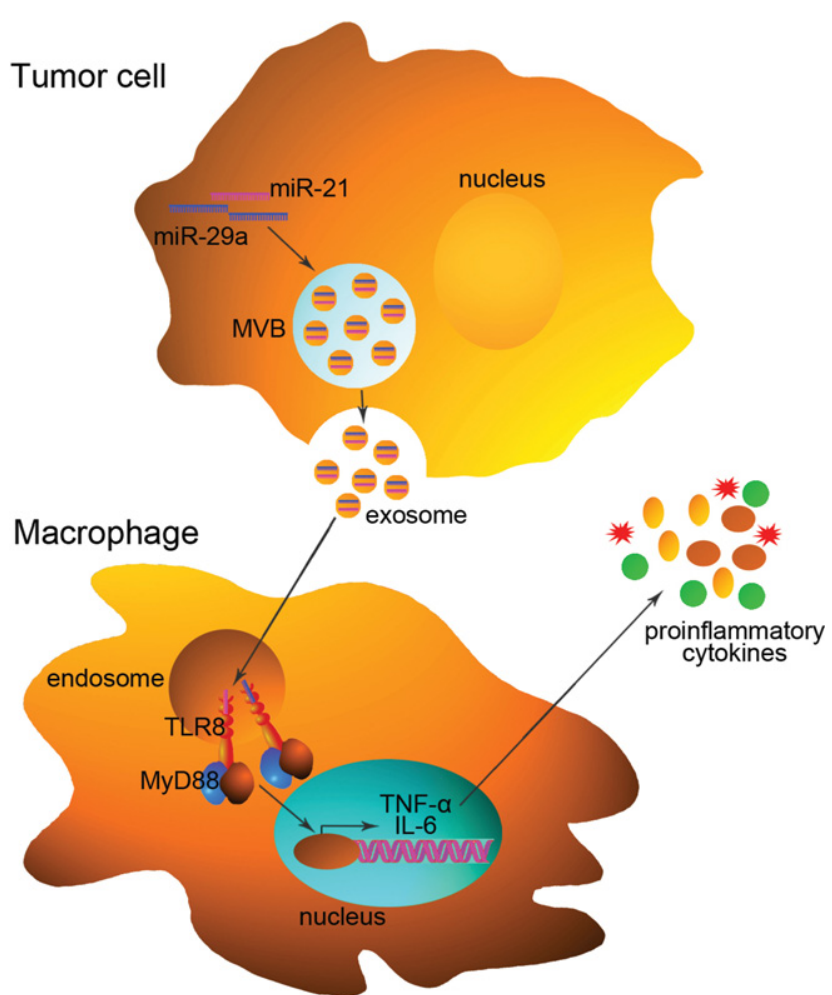

FIGURE 1. Recognition of miRNAs by TLRs in tumor cells. miRNAs of tumor cells, such as miR-21 and miR-29a, can be packaged into exosomes and secreted to the extracellular environment. Exosomes can transfer these miRNAs to recipient macrophages and eventually reach endosomes, in which miR-21 and miR-29a are recognized by endosomally expressed human TLR8 (or murine TLR7). After the ligation of TLR8 (or TLR7), the adaptor molecule MyD88 is recruited and drives the production of proinflammatory cytokines such as TNF- $\alpha$ and IL-6. This pathway may ultimately lead to tumor growth and metastasis.

to tumor growth and metastasis (Fabbri et al. 2012). Thus, by acting as paracrine ligands of TLRs, miRNAs are key regulators of the tumor microenvironment. This mechanism of action of miRNAs is implicated in tumor-immune system communication and is important in tumor growth and spread, thus representing a possible target for cancer treatment.

\section{miRNAs FUNCTION AS TLR LIGANDS IN NEURONS}

Lehmann et al. found that let- $7 \mathrm{~b}$ can act as a potent activator of TLR7 signaling in neurons and that this activation can induce neurodegeneration; thus, their experiments revealed a new role of miRNAs beyond their function as regulators of gene expression (Fig. 2). Specifically, Lehmann et al. postulated that let-7b can act as a signaling activator of TLR7 because the let-7b sequence contains a core GU-rich motif that is also present in the HIV ssRNA40, which is a known ligand of TLR7 (Heil et al. 2004). In agreement with this hypothesis, synthetic let-7b activates the release of TNF- $\alpha$ from microglia and mac- rophages through TLR7 (Lehmann et al. 2012). Furthermore, the exposure of neurons to extracellular let-7b was found to induce neurodegeneration through the activation of TLR7, but $\mathrm{Tlr}^{-1-}$ neurons were resistant to let-7b (Lehmann et al. 2012). Subsequently, Lehmann et al. hypothesized that let$7 \mathrm{~b}$ might be released during neuronal injury to act on neighboring neurons, which, in turn, would accelerate neuronal damage when these neurons release neurotoxic let-7b. To test this hypothesis, these researchers analyzed whether endogenous let- $7 \mathrm{~b}$ released from dying neurons injures neurons in a manner similar to that of synthetic let-7b and whether this effect can be blocked by let-7b antagonists. As expected, they showed that the addition of culture supernatants from apoptotic or necrotic neurons (in which high levels of let-7b were detected) to fresh primary neurons induced injury and loss, an effect that could be abrogated by pretreatment of the neurons with a let-7b inhibitor (Lehmann et al. 2012). Lehmann et al. then evaluated the role of let- $7 b$ as an activator of TLR7 in neurons in vivo. The intrathecal injection of let-7b into mice induced marked axonal injury and neuronal loss, and these effects were blocked by pretreatment with a let-7b inhibitor (Lehmann et al. 2012). Finally, these researchers

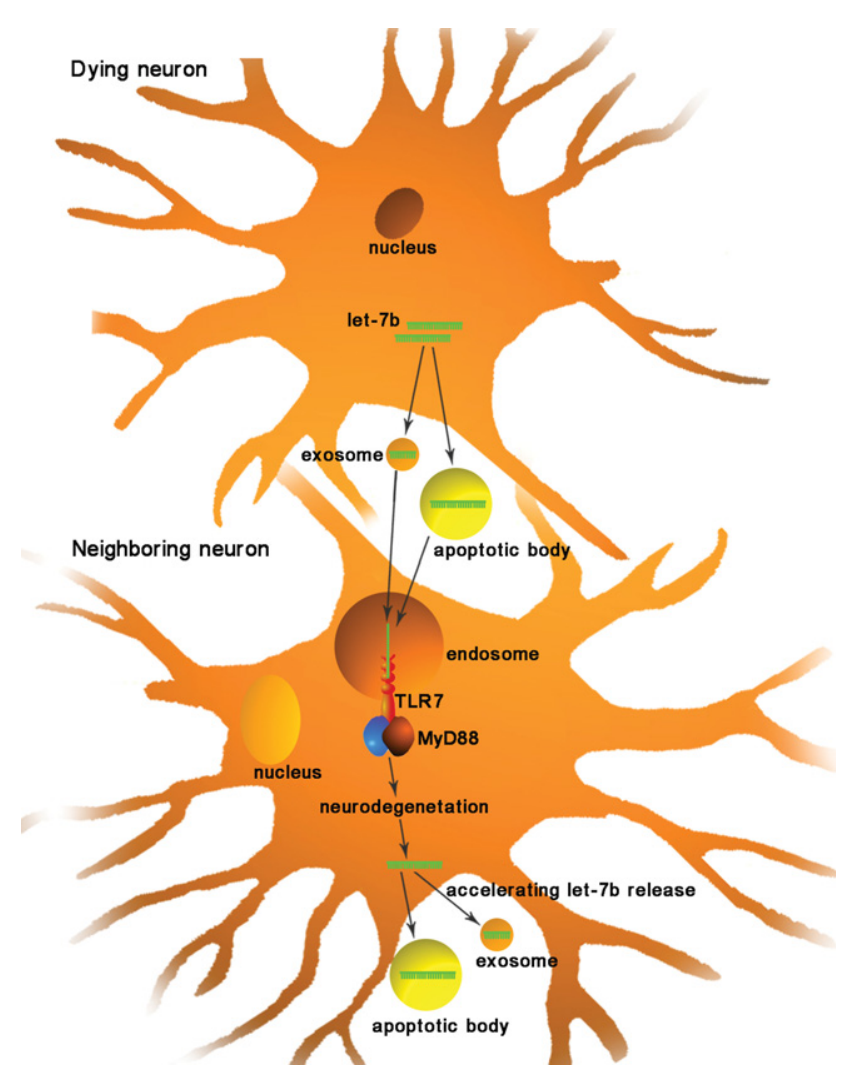

FIGURE 2. Recognition of miRNAs by TLRs in neurons. During neuronal injury, let-7b is released from dying neurons through apoptotic bodies or exosomes (the exact mechanism remains to be elucidated). Exogenous let-7b can act as an activator of TLR7 in neighboring neurons, thereby sending a damage signal to neurons and accelerating neurodegeneration. 
detected elevated let-7b levels in the cerebrospinal fluid of Alzheimer's patients (Lehmann et al. 2012). Together, although the interaction of let-7b and murine TLR7 has been indirectly demonstrated without ever performing coimmunoprecipitation experiments, the results revealed a new role of miRNAs as endogenous activators of TLRs in neurons. The proposed mechanism of this signaling pathway is that let-7b released under pathological conditions stimulates TLR7, thereby sending a danger signal to neurons and accelerating their decay.

\section{BIOLOGICAL RELEVANCE OF miRNAs AS TLR LIGANDS}

The discovery of this new function of miRNAs raises some fascinating questions. First, what is the underlying mechanism through which miRNAs bind to intracellular TLRs? According to the current results, the GU-rich motif (GUUG for miR-21, GGUU for miR-29a, and GUUGUGU for let$7 b$ ) is essential for the miRNA-TLR recognition. Thus, individual miRNAs may contain important information that allows selective access of these miRNAs to TLRs. However, other questions remain. Do other structural features in the sequences of miRNAs confer the capacity to activate TLRs? Can TLRs other than murine TLR7 and human TLR8 be recognized by miRNAs? The core element guiding the miRNATLR interaction remains to be determined. Second, why do some miRNAs have dual activities? It has long been known that miR-21, miR-29a, and let-7b play important biological roles in cells through classic post-transcriptional regulation (Meng et al. 2007; Zhao et al. 2010). Therefore, these miRNAs have dual functions: On the one hand, they bind to Argonaute proteins and guide the silencing of target genes, and on the other hand, they act independently of Argonaute proteins by interacting directly with TLRs. Are these miRNAs first released from Argonaute proteins and then bound by TLRs? How is the interplay between the two pathways regulated? Further investigations are required to assess the biological significance of the dual role of these miRNAs. Finally, what is the impact of miRNAs acting as TLR ligands in the pathogenesis of diseases other than cancer and neurodegeneration? Future studies will be necessary to unravel the mechanistic details underlying the diverse functions of these miRNAs in both healthy and disease states.

In summary, miRNAs not only repress protein expression at the post-transcriptional level but also serve as ligands of TLRs. Although many fundamental questions remain to be answered, the discovery of this new role of miRNAs opens a new and exciting field of miRNA research.

\section{ACKNOWLEDGMENTS}

This work was supported by grants from the National Natural Science Foundation of China (No. 81101330, 31271378, 81250044, and J1103512) and the Natural Science Foundation of Jiangsu Province (No. BK2011013 and BK2012014).

\section{REFERENCES}

Bartel DP. 2004. MicroRNAs: Genomics, biogenesis, mechanism, and function. Cell 116: 281-297.

Blasius AL, Beutler B. 2010. Intracellular toll-like receptors. Immunity 32: 305-315.

Chen X, Liang H, Zhang CY, Zen K. 2012a. miRNA regulates noncoding RNA: A noncanonical function model. Trends Biochem Sci 37: 457459.

Chen X, Liang H, Zhang J, Zen K, Zhang CY. 2012b. Secreted microRNAs: A new form of intercellular communication. Trends Cell Biol 22: 125-132.

Fabbri M, Paone A, Calore F, Galli R, Gaudio E, Santhanam R, Lovat F, Fadda P, Mao C, Nuovo GJ, et al. 2012. MicroRNAs bind to Toll-like receptors to induce prometastatic inflammatory response. Proc Natl Acad Sci 109: E2110-E2116.

Heil F, Hemmi H, Hochrein H, Ampenberger F, Kirschning C, Akira S, Lipford G, Wagner H, Bauer S. 2004. Species-specific recognition of single-stranded RNA via Toll-like receptor 7 and 8. Science 303: $1526-1529$.

Lehmann SM, Kruger C, Park B, Derkow K, Rosenberger K, Baumgart J, Trimbuch T, Eom G, Hinz M, Kaul D, et al. 2012. An unconventional role for miRNA: let- 7 activates Toll-like receptor 7 and causes neurodegeneration. Nat Neurosci 15: 827-835.

Meng F, Henson R, Wehbe-Janek H, Ghoshal K, Jacob ST, Patel T. 2007. MicroRNA-21 regulates expression of the PTEN tumor suppressor gene in human hepatocellular cancer. Gastroenterology 133: 647658.

Takeda K, Kaisho T, Akira S. 2003. Toll-like receptors. Annu RevImmunol 21: 335-376.

Zhao C, Sun G, Li S, Lang MF, Yang S, Li W, Shi Y. 2010. MicroRNA let$7 b$ regulates neural stem cell proliferation and differentiation by targeting nuclear receptor TLX signaling. Proc Natl Acad Sci 107: 18761881. 

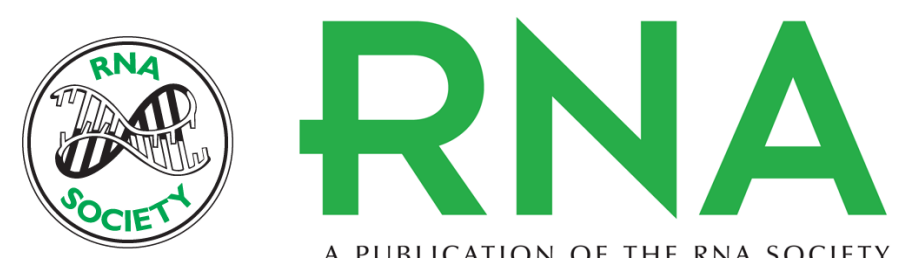

A PUBLICATION OF THE RNA SOCIETY

\section{microRNAs are ligands of Toll-like receptors}

Xi Chen, Hongwei Liang, Junfeng Zhang, et al.

RNA 2013 19: 737-739 originally published online April 3, 2013

Access the most recent version at doi:10.1261/rna.036319.112

$\begin{array}{ll}\text { References } & \begin{array}{l}\text { This article cites } 10 \text { articles, } 3 \text { of which can be accessed free at: } \\ \text { http://rnajournal.cshlp.org/content/19/6/737.full.html\#ref-list-1 }\end{array}\end{array}$

\section{License}

Email Alerting Receive free email alerts when new articles cite this article - sign up in the box at the Service top right corner of the article or click here.

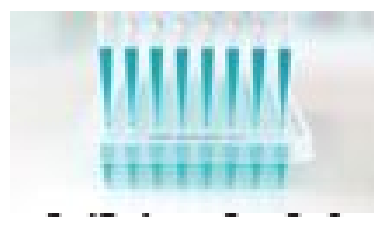

\section{Providing Precise Solutions for} your research.

To subscribe to RNA go to:

http://rnajournal.cshlp.org/subscriptions 